Original paper

\title{
Digital Imaging and Communications in Medicine (DICOM) information conversion procedure for SUV calculation of PET scanners with different DICOM header information
}

\author{
Han-Back Shin ${ }^{a}$, Heesoon Sheen ${ }^{\mathrm{b}, \mathrm{c}}$, Ho-Young Lee ${ }^{\mathrm{d}}$, Jihoon Kang ${ }^{\mathrm{e}}$, Do-Kun Yoon ${ }^{\mathrm{a}, *}$, Tae Suk Suh ${ }^{\mathrm{a}, *}$ \\ a Department of Biomedical Engineering and Research Institute of Biomedical Engineering, College of Medicine, The Catholic University of Korea, Seoul 06591, Republic of Korea \\ ${ }^{\mathrm{b}}$ School of Medicine, Sungkyunkwan University, \#50 Ilwon-dong, Kangnam-ku, Seoul 135-710, Republic of Korea \\ ${ }^{c}$ GE Healthcare Korea, Gheongdam-dong, Gangnam-ku, Seoul 135-100, Republic of Korea \\ ${ }^{\mathrm{d}}$ Department of Nuclear Medicine, Seoul National University Bundang Hospital, Seongnam 13620, Republic of Korea \\ e Department of Biomedical Engineering, Chonnam National University, Yeosu 59626, Republic of Korea
}

\section{A R T I C L E I N F O}

\section{Article history:}

Received 2 February 2017

Received in Revised form 17 April 2017

Accepted 20 May 2017

Available online 24 May 2017

\section{Keywords:}

DICOM

SUV

PET

\begin{abstract}
A B S T R A C T
Purpose: In nuclear medicine, the standardized uptake value (SUV) obtained using positron emission tomography with 2-deoxy-2-fluoro-D-glucose (FDG-PET) is widely used as a semi-quantitative diagnosis factor. We found that the header file of the Philips Allegro PET scanner using the Digital Imaging and Communications in Medicine (DICOM) standard was stored differently than with other scanners. Thus, the purpose of this study was to develop a DICOM header information conversion program to ensure compatibility between Allegro and other equipment.

Methods and results: The NEMA IEC Body phantom was scanned using the Allegro PET scanner. We conducted measurements and performed calculations by using commercial software and the proposed selfdeveloped program, respectively, to compare the SUVs by using conversion data. The program consists of three parts: an input part that can load data regardless of the number of DICOM images, and conversion and output parts that can be used to convert the DICOM header information and store it in the order of slices. The results of the calculation are in good agreement with the data measured at 12 circular regions of interest. The percent difference was lower than the $20 \%$.

Conclusion: In conclusion, this study suggested a simple and convenient method to solve the incompatibility through conversion of the DICOM header information. This study thus provides physicians more accurate information for diagnosis and treatment.
\end{abstract}

(c) 2017 Published by Elsevier Ltd on behalf of Associazione Italiana di Fisica Medica.

\section{Introduction}

In nuclear medicine, positron emission tomography (PET) scanners are widely used for lesion detection [1-5]. In particular, the PET scan with glucose analogue $2-\left[{ }^{18}\right.$ fluorine]-fluoro-2-deoxy-dglucose $\left({ }^{18} \mathrm{~F}-\mathrm{FDG}\right)$ has emerged as an important method to evaluate the presence or absence of cancer, its staging, and prognosis in accordance with the characteristics of the FDG [6,7].

The standardized uptake value (SUV) is commonly used in FDG studies as a semi-quantitative factor that can be obtained from PET images [8-10]. Although the SUV has uncertainties in the region of interest (ROI) or subject size, it has become one of the most

\footnotetext{
* Corresponding authors.

E-mail addresses: dbsehrns@catholic.ac.kr (D.-K. Yoon), suhsanta@catholic.ac.kr (T.S. Suh).
}

important factors for the lesion detectability, owing to its usefulness, as demonstrated in many studies [8-13]. The SUV is derived as the ratio of the dose radioactivity at scan time and at the injected time according to the patient's body weight. The SUV can be calculated using the following equations [8,10,14-16]:

$$
\mathrm{SUV}_{\text {body weight }\left(\frac{\mathrm{kg}}{\mathrm{cc}}\right)}=\frac{\text { Activity Concentrationin } R O I_{\left(\frac{B q}{c c}\right)}}{\left(\frac{\text { Injected Dose }(B q)}{\text { Body weight }(\mathrm{kg})}\right)}
$$

where, Activity Concentration is the activity per volume of the ROI at the image acquisition time, and the Injected Dose is the dose at injection time. [8,10,14-16].

$$
\mathrm{SUV}_{\text {body weight }\left(\frac{\mathrm{kg}}{\mathrm{cc}}\right)}=\frac{(\text { Pixel value } \times \text { Image Rescale factor } \times \text { dosecalibration })}{\left(\frac{\text { Actual activity }}{\text { Body wight }}\right)}
$$


where Pixel Value is the pixel intensity value in the ROI, the Image Rescale Factor indicates the conversion factor for conversion pixel value to $\mathrm{Bq}$, the Dose Calibration factor is a correction factor from $\mathrm{MBq} / \mathrm{cc}$ to $\mathrm{Bq} / \mathrm{cc}$, and the Actual Activity is that of the image acquisition time $[10,16]$. The factors necessary for calculating the SUV should be determined for use in the unified Digital Imaging and Communications in Medicine (DICOM) standard. Since the first version of DICOM standard was published by the American College of Radiology and National Electrical Manufacturers Association, the contents of the DICOM standard have been modified and improved [17]. The DICOM file is composed of a medical image and a header file that contain information including image parameters, and this information includes interoperability between medical information systems $[17,18]$. However, if a manufacturer does not follow the DICOM standard, then there is no compatibility between products, and this can lead physicians to make incorrect assessments when they diagnose diseases by using PET imaging. We found that the DICOM header file of the Philips Allegro PET scanner (Philips Medical Systems; Milpitas, CA, USA) that includes the factors for SUV calculation was stored differently when compared to General Electric (GE) and Siemens equipment. Thus, the purpose of this study was to develop a DICOM information conversion program to ensure compatibility between the Allegro and other equipment. For this purpose, we measured the SUVs before and after DICOM conversion and compared them to the calculated value.

\section{Methods and Materials}

\subsection{Phantom preparation}

The National Electrical Manufacturers Association(NEMA) International Electrotechnical Commission (IEC) Body Phantom was scanned using the Philips Allegro PET scanner [19]. The NEMA IEC Body phantom is widely recommended for evaluation of whole-body PET imaging. As this phantom had 6 spherical phantoms with different diameter, we set the 6 ROIs on the spheres and 6 ROIs on the background region. These spherical phantoms had inner diameter of $10,13,17,22,28$, and $37 \mathrm{~mm}$, respectively. The size of ROI was set as $70 \%$ of sphere's physical inner diameter. The activity ratio between sphere and background area is $4: 1$ based on the NEMA NU 2-2007 standard [20]. Data were acquired in 3D mode. The data were reconstructed in a $144 \times 144$ matrix with a slice thickness of $4 \mathrm{~mm}$. For the image reconstruction, the 3D RAMLA reconstruction algorithm was applied to phantom datasets. The effective axial field of view was $14.8 \mathrm{~cm}$.

The phantom was filled with $56 \mathrm{MBq}$ of ${ }^{18} \mathrm{~F}$, and the weight of the phantom was $12 \mathrm{~kg}$ including the radiopharmaceuticals. We measured the SUVs by using commercially-available software (GE advantage 4.6 Workstation, GE Healthcare, Milwaukee, WI, USA) and calculated the SUVs by using MATLAB (Mathworks Inc., Sherborn, MA, USA) for comparison.

\subsection{DICOM header information conversion and SUV calculation}

To solve the problem of the incompatibility between products, we developed a program based on self-developed MATLAB code (R2014a, Mathworks, Inc., MI). The primary reason for the incompatibility between the Philips Allegro and GE and SIEMENS equipment is due to the different units used to present the image information. The Allegro uses the counts (CNTS) as units, but the other manufacturers use Bq/cc. These differences result an incompatibility in reading the Allegro data by using GE's commercial software for the SUV. As shown in Fig. 1, the data obtained from the PET scanners of different companies can be processed by their respective software. However, if they are incompatible, the process of SUV measurement may not be possible. The proposed system is compatible with the non-compatible parts through the DICOM header information conversion.

According to the DICOM technical report for the Allegro and the Gemini System, the additional private data element does not conform to standard PET information object definition [19]. Among such private data, the related attributed tag, which can identify the element as private data $(7053,1000)$, is used to convert from counts to SUV. However, because this information is the conversion factor per selected slice and not for the ROI, it is not helpful at all when specifying the ROI. Thus, the conversion program is useful and essential for measuring the SUV within the ROI. Eq. (3) was used to convert the units from intensity to activity (Bq/ cc) within the ROI.

Activity Concentration $\left(\frac{B q}{c c}\right)=\alpha X+\beta$

where $\alpha$ is the rescale slope (0028, 1053), $X$ is the original pixel intensity, and $\beta$ is the rescale intercept $(0028,1052)$. Because $\alpha$ and $\beta$ have different values for each slice, the calculated Activity Concentration is the activity concentration on the ROI at the selected slice. In addition, the conversion program was composed of input, conversion and output stages, as shown in Fig 2 . The input stage can be loaded regardless of the number of the DICOM images, and the conversion and output stages convert the DICOM header information and store it in the order of the slices. The key function and precautions which we used in the MATLAB code are as follows.

Input stage: we used the function 'dicomread' for the DICOM image file input and 'dicominfo' for the DICOM header information. In this procedure, since the data may not be input in the order of slice, it is essential to sort based on the image index, which indicates the slice number known through 'dicominfo'. The precautions for this process is to use the 'fileparts' function to determine the required data format such as DICOM. During the conversion stage, as stated in this section, factors for SUV calculation can substitute and compute the values in the DICOM header information, such that the user can view the factors using the 'dicominfo' function. Output stage: To merge the DICOM image file and the header information, we use the 'dicomwrite' function. To do this, the image file and the converted DICOM header information are entered as the input factor of 'dicomwrite', and the file name is saved as specified by the user.

\section{Results and discussion}

Fig. 3 indicated the DICOM header information shown on MATLAB window. With this process, it is possible to use the MATLAB internal function such as dicominfo('filename'). As shown in Fig 3, the DICOM header information consisted of the factors based on the DICOM standard and the private factors depended on the company or the device. Therefore, if users hope to access and convert the DICOM data, it is important to know the meaning of these factors. Fig. 4 shows the NEMA IEC Body phantom image acquired using the Philips Allegro equipment. Commercial software (GE advantage 4.6 Workstation, GE Healthcare, Buckinghamshire, UK) was used to measure the SUVs on 12 circular ROIs, as shown in Fig. 4(a). To verify the conversion process and calculate the SUVs, ImageJ (Rasband, W.S., U. S. National Institutes of Health, Bethesda) and MATLAB code were used to select the ROI and calculate the SUVs. To confirm that the converted data was functioning normally, we measured the SUVs using the commercial software and calculated the SUVs using Eqs. (1) or (2). However, the converted activity values from the pixel intensity do not consider the acquisition time, scan time, and the radiation decay time. To take these values of time into consideration, the decay correc- 

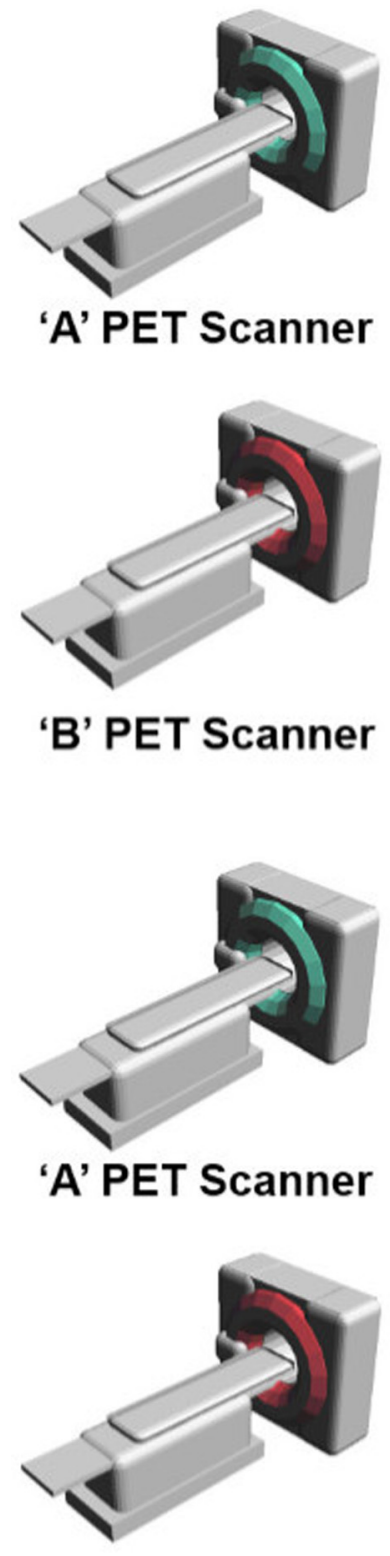

'B' PET Scanner

\section{Conventional System}

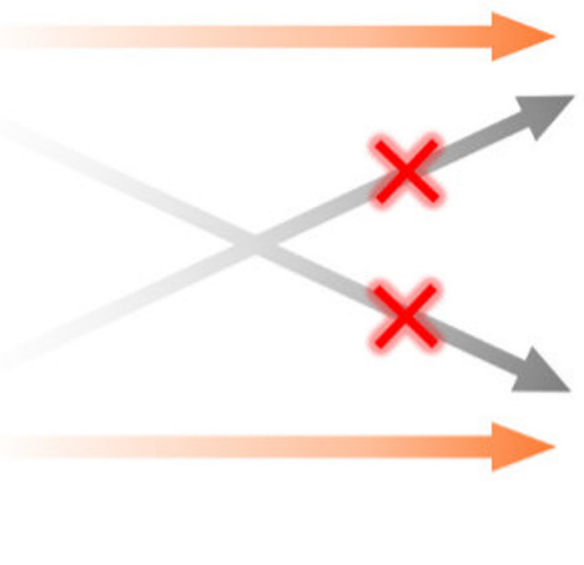

'B' Software
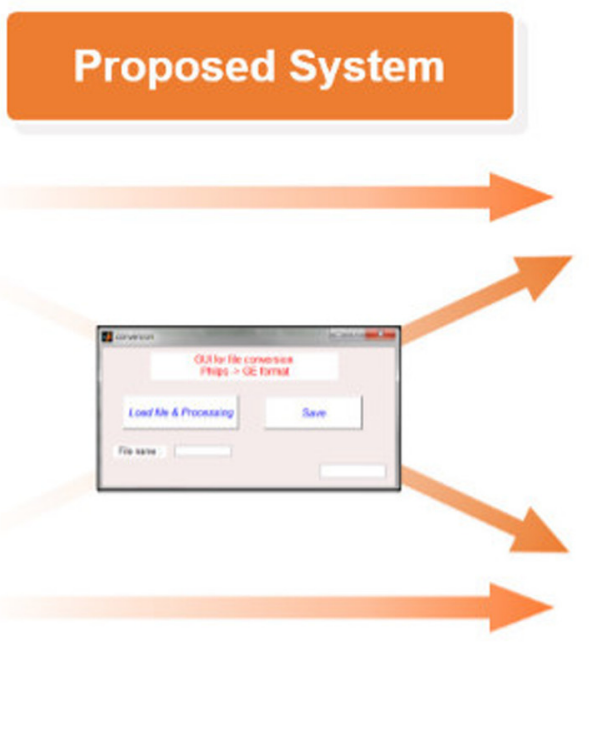

'B' Software

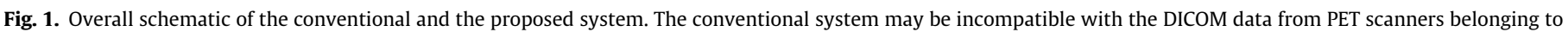
different companies, whereas the proposed system is compatible with the DICOM data stored through the DICOM header information conversion.

tion should be applied in order to calculate the actual activity at scan time. The related tags in this correction procedure are the radionuclide total dose $(0018,1074)$, series data $(0008,0021)$, and radiopharmaceutical half time $(0018,1075)$. These tags were utilized to calculate the actual activity reflected as in the following equations $[8,10,14-16]$ :

Injected dose at a scantime $(B q)=D(B q) \times$ Decay ratio

Decay ratio $=e^{\left(-\log (2)\left(\frac{t 1-t 2}{\text { half life time }}\right)\right)}$

where $D$ is the radionuclide total dose $(0018,1074), t_{1}$ is the series time $(0008,0031), t_{2}$ is the radiophamaceutical start time $(0018$, 1072), and half-life time is the radiophamaceutical half time $(0018,1075)$.
Table 1 presents the measured data and calculated data using the converted DICOM image. As shown in Table 1, the measured data on twelve ROIs were compared to the calculated data, showing the difference in percentage; the maximum difference was less than $20 \%$. We conjectured that the reason of this difference is the difference in size of the ROI of the commercial software (GE advanced workstation) and Image because we use the pixel intensity value obtained from the commercial software, we confirmed that the SUV are the same when the commercial software and our method are processed. We then confirmed that the converted DICOM file obtained using the conversion program could normally calculate the SUVs. Previous studies have shown that SUV can be changed by body weight, image reconstruction, and the correction method $[8,11,16]$. Because these studies suggested that SUV has uncertainties, the measurement method and correction method are important for performing diagnosis using the SUV. In addition, 


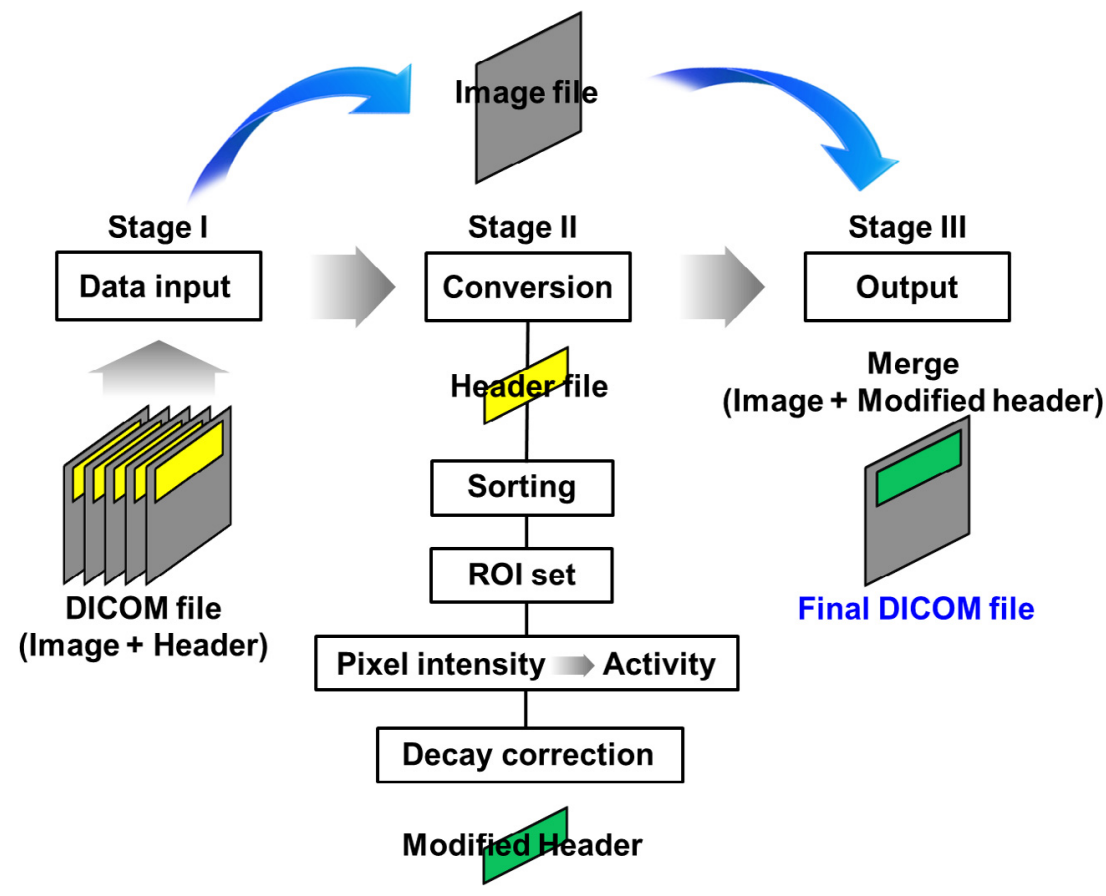

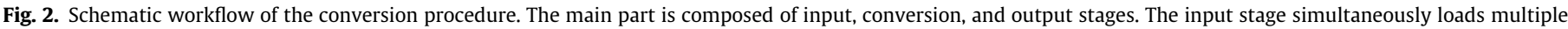

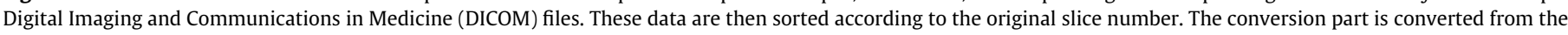

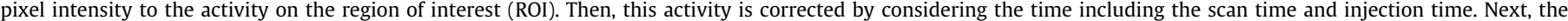
output stage writes the DICOM file, including conversion DICOM header information, and the original image.

(a)

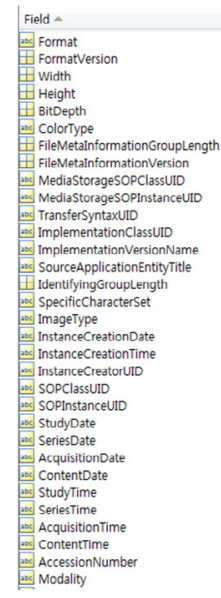

(b)

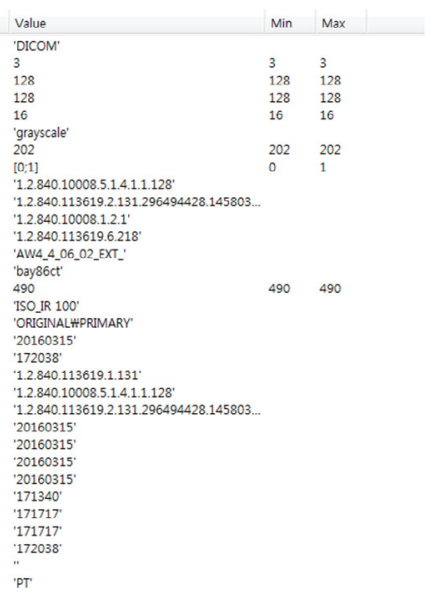

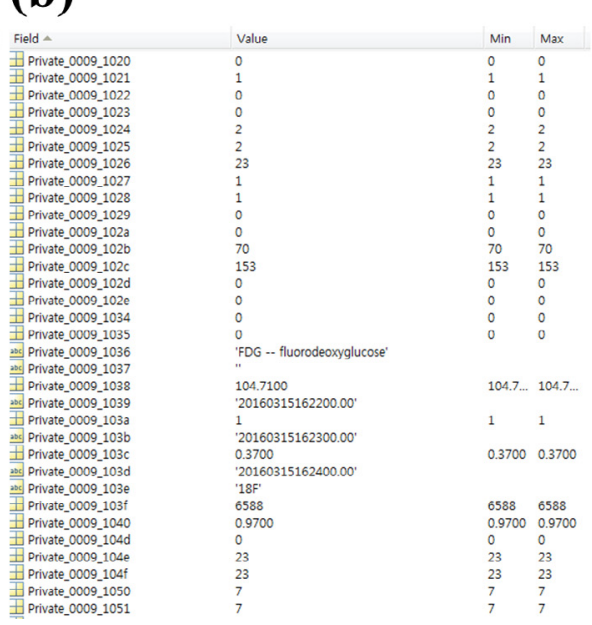

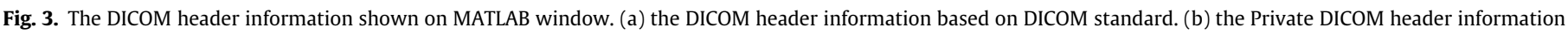
depend on company or device.

since the SUVs are calculated by factors in the DICOM header information, the SUV measurement can be performed by using the DICOM header information $[10,18]$. Our study proposed and demonstrated that the DICOM header information conversion can be used to solve problems such as incompatibility among different scanners caused by different DICOM header information. The method proposed in this study could be applied to estimate the results by using the conversion of the DICOM header information. However, because this study specifically deals with the incompatibility between scanners having different DICOM header information, while applying it to other research problems, the subject of research should be carefully assessed. It can be used by applying the approach and conversion method for DICOM header information as shown in Figs. 2 and 3.

\section{Conclusion}

In conclusion, we found that the incompatibility is due to the differences in the DICOM header information. This study suggested a simple and convenient method to solve the incompatibility. Moreover, we demonstrated that the self-developed DICOM header conversion program enables compatibility with other equipment, and we showed the detailed conversion procedure. The results of 
(a)

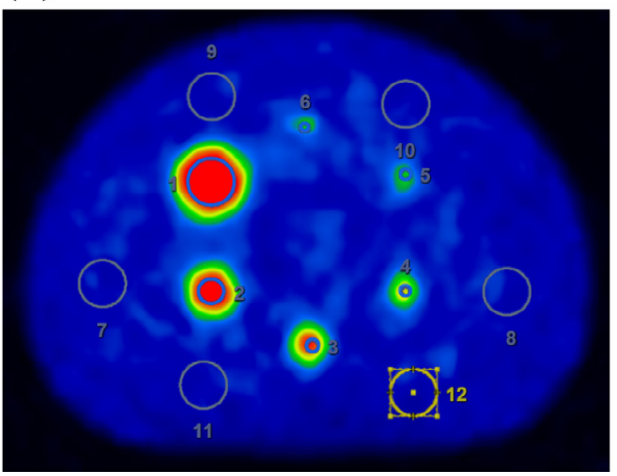

(b)

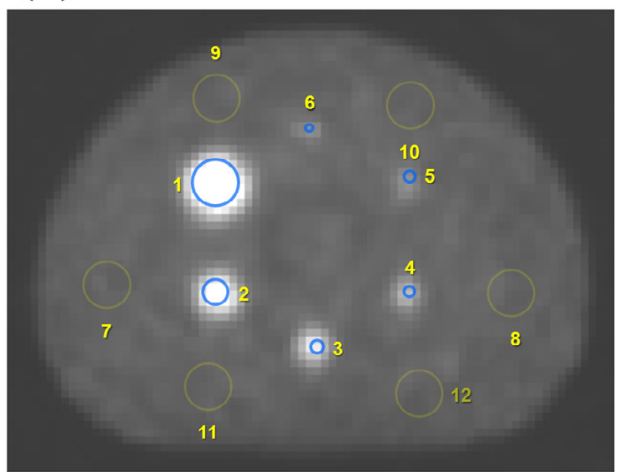

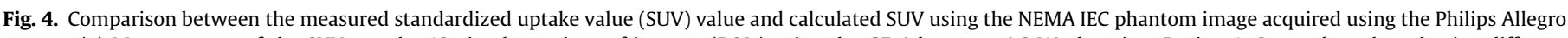

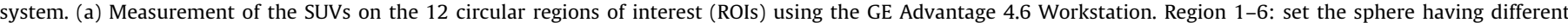

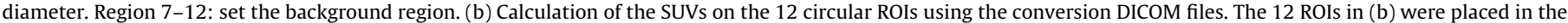
similar position as those in (a) with having similar diameter.

Table 1

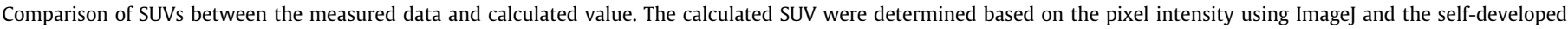
program with 12 circular ROIs. The values of the SUV were measured based on Bq/cc (conversion data) using commercial software (GE Advanced Workstation).

\begin{tabular}{|c|c|c|c|}
\hline Regions & Measurement value (Average $\pm S D$ ) & Calculation value(Average \pm SD) & Percent difference $(\%)\left(\left|\frac{\text { Measurement value Calculation value }}{\text { Measurement value }}\right| \times 100\right)$ \\
\hline Region 1 & $6.13 \pm 0.6$ & $6.27 \pm 0.50$ & 28 \\
\hline Region 2 & $5.82 \pm 0.52$ & $5.85 \pm 0.44$ & 0.43 \\
\hline Region 3 & $4.47 \pm 0.54$ & $4.92 \pm 0.03$ & 10.01 \\
\hline Region 4 & $3.44 \pm 0.21$ & $3.86 \pm 0.55$ & 12.22 \\
\hline Region 5 & $2.25 \pm 0.16$ & $2.70 \pm 0.25$ & 20.11 \\
\hline Region 6 & $2.21 \pm 0.08$ & $2.23 \pm 0.05$ & 1.01 \\
\hline Region 7 & $1.08 \pm 0.08$ & $1.08 \pm 0.07$ & 0.08 \\
\hline Region 8 & $1.1 \pm 0.13$ & $1.06 \pm 0.10$ & 3.26 \\
\hline Region 9 & $1.09 \pm 0.08$ & $1.07 \pm 0.09$ & 2.06 \\
\hline Region 10 & $1.08 \pm 0.07$ & $1.08 \pm 0.07$ & 0.46 \\
\hline Region 11 & $1.07 \pm 0.08$ & $1.07 \pm 0.10$ & 0.65 \\
\hline Region 12 & $1.01 \pm 0.13$ & $1.12 \pm 0.10$ & 10.65 \\
\hline
\end{tabular}

this study will provide physicians with more accurate information for diagnosis and treatment. In addition, we expect that this study will give a way to solve the incompatibility between such products by properly managing DICOM information. However, our results show that the percent difference of the SUV could be caused by the ROI, as shown in Table 1. Therefore, it should always be noted that quantitative analysis of nuclear medicine images is more variable with uncertainties because there are uncertainties in the SUV, due to ROI, software, and the correction method. In future work, we plan to develop and apply a unified DICOM conversion software for big data analysis.

\section{Conflict of interest}

None.

\section{Acknowledgments}

This work was supported by the Mid-career Researcher Program (2014R1A2A1A10050270) through the National research Foundation of Korea funded by the Ministry of Science, ICT \& Future Planning, Republic of Korea.

\section{References}

[1] Frackowiak RS, Lenzi G-L, Jones T, Heather JD. Quantitative measurement of regional cerebral blood flow and oxygen metabolism in man using 150 and positron emission tomography: theory, procedure, and normal values. J Comput Assist Tomogr 1980;4:727-36.
[2] Wong DF, Wagner HN, Tune LE, Dannals RF, Pearlson GD, Links JM, et al Positron emission tomography reveals elevated D2 dopamine receptors in drug-naive schizophrenics. Science 1986;234:1558-63.

[3] Chugani HT, Phelps ME, Mazziotta JC. Positron emission tomography study of human brain functional development. Ann Neurol 1987;22:487-97.

[4] Schiepers C, Penninckx F, De Vadder N, Merckx E, Mortelmans L, Bormans G, et al. Contribution of PET in the diagnosis of recurrent colorectal cancer: comparison with conventional imaging. Eur J Surg Oncol 1995;21:517-22.

[5] Shin HB, Choi Y, Huh Y, Jung J, Suh T. Conceptual Study of Brain Dedicated PET Improving Sensitivity. Prog Med Phys 2016;27:236-40.

[6] Adams S, Baum RP, Stuckensen T, Bitter K, Hor G. Prospective comparison of 18F-FDG PET with conventional imaging modalities (CT, MRI, US) in lymph node staging of head and neck cancer. Eur J Nucl Med 1998;25:1255-60.

[7] Mosconi L, Tsui WH, Herholz K, Pupi A, Drzezga A, Lucignani G, et al. Multicenter standardized 18F-FDG PET diagnosis of mild cognitive impairment, Alzheimer's disease, and other dementias. J Nucl Med 2008;49:390-8.

[8] Zasadny KR, Wahl RL. Standardized uptake values of normal tissues at PET with 2-[fluorine-18]-fluoro-2-deoxy-D-glucose: variations with body weight and a method for correction. Radiology 1993;189:847-50.

[9] Torizuka T, Tamaki N, Inokuma T, Magata Y, Yonekura Y, Tanaka A, et al. Value of fluorine-18-FDG-PET to monitor hepatocellular carcinoma after interventional therapy. J Nucl Med 1994;35:1965-9.

[10] Masa-Ah P, Tuntawiroon M, Soongsathitanon S. A novel scheme for Standardized Uptake Value (SUV) calculation in PET scans. MMMAS 2010;4:291-9.

[11] Matheoud R, Ferrando O, Valzano S, Lizio D, Sacchetti G, Ciarmiello A, et al. Performance comparison of two resolution modeling PET reconstruction algorithms in terms of physical figures of merit used in quantitative imaging. Phys Med 2015;31(5):468-75.

[12] Chirla R, Marcu LG. PET-based quantification of statistical properties of hypoxic tumor subvolumes in head and neck cancer. Phys Med 2016;32 (1):23-35.

[13] Chauvie Stephane, Bergesio Fabrizio, Fioroni Federica, Brambilla Marco, Biggi Alberto, Versari Annibale, et al. The 68Ge phantom-based FDG-PET site qualification program for clinical trials adopted by FIL (Italian Foundation on Lymphoma). Phys Med 2016;32(5):651-6. 
[14] Boland GW, Goldberg MA, Lee MJ, Mayo-Smith WW, Dixon J, McNicholas MM, et al. Indeterminate adrenal mass in patients with cancer: evaluation at PET with 2-[F-18]-fluoro-2-deoxy-D-glucose. Radiology 1995;194:131-4.

[15] Inoue T, Kim EE, Wong FC, Yang DJ, Bassa P, Wong WH, et al. Comparison of fluorine-18-fluorodeoxyglucose and carbon-11-methionine PET in detection of malignant tumors. J Nucl Med 1996;37:1472-6.

[16] Pierce 2nd LA, Elston BF, Clunie DA, Nelson D, Kinahan PE. A Digital Reference Object to Analyze Calculation Accuracy of PET Standardized Uptake Value. Radiology 2015;277:538-45.

[17] Mildenberger P, Eichelberg M, Martin E. Introduction to the DICOM standard. Eur Radiol 2001;12:920-7.
[18] Bidgood WD, Horii SC, Prior FW, Van Syckle DE. Understanding and Using DICOM, the Data Interchange Standard for Biomedical Imaging. J Am Med Inform Assoc 1997;4:199-212.

[19] Daouk J, Fin L, Bailly P, Meyer ME. Improved attenuation correction via appropriate selection of respiratory-correlated PET data. Comput Methods Programs Biomed 2008;92:90-8.

[20] NEMA Standards Publication NU 2-2007. Performance Measurements of Positron Emission Tomographs. Waszyngton: National Electrica Manufacturers Association; 2007. 